\title{
Collective dynamics of a network of ratchets coupled via a stochastic dynamical environment
}

\author{
U. E. Vincent, ${ }^{1,2, *}$ B. R. Nana-Nbendjo, ${ }^{3,4}$ and P. V. E. McClintock ${ }^{1}$ \\ ${ }^{1}$ Department of Physics, Lancaster University, Lancaster LAI 4YB, United Kingdom \\ ${ }^{2}$ Department of Physical Sciences, Redeemer's University, Km 46, Lagos-Ibadan Expressway, P.M.B 3005, Redemption City, Nigeria \\ ${ }^{3}$ Laboratory of Modeling and Simulation in Engineering and Biological Physics, Faculty of Sciences, University of Yaoundé I, \\ PO Box 812 Yaoundé, Cameroon \\ ${ }^{4}$ Max-Planck-Institut für Physik Komplexer Systeme Nöthnitzer Strasse 38, D-01187 Dresden, Germany
}

(Received 27 February 2012; published 22 February 2013)

\begin{abstract}
We investigate the collective dynamics of a network of inertia particles diffusing in a ratchet potential and interacting indirectly through their stochastic dynamical environment. We obtain analytically the condition for the existence of a stable collective state, and we show that the number $N$ of particles in the network, and the strength $k$ of their interaction with the environment, play key roles in synchronization and transport processes. Synchronization is preceded by symmetry-breaking associated with double-resonance oscillations and is shown to be strongly dependent on the network size: convergence to the synchronization manifold occurs much faster with a large network. For small networks, increasing the noise level enhances synchronization in the weakly coupled regime, while particles in a large network are weakly synchronized. Similarly, in the strongly coupled regime, particles in a small network are weakly synchronized; whereas the synchronization is strong and robust against noise when the network-size is large. Small and moderate networks maximize and stabilize efficient transport. Although the dynamics for larger networks is highly correlated, the transport current is erratic.
\end{abstract}

DOI: 10.1103/PhysRevE.87.022913 PACS number(s): 05.45.Xt, 05.40.Fb, 05.45.Pq, 05.60.Cd

\section{INTRODUCTION}

Noisy dynamics driven by thermal fluctuations is the hallmark of many microscopic systems. Where there is broken symmetry, as in Brownian ratchets, the noise may be utilized through the conversion of nonequilibrium fluctuations into directed motion in the absence of imposed bias forces [1-4]. Understanding and generating directed transport in nonequilibrium systems has long been considered a problem of fundamental importance in statistical physics and has recently been the subject of strongly focused research [1-4]. In fact, the driving mechanisms of a variety of biological motors [2,3], ranging from intercell transport and virus translocation to muscle contraction, are all traceable to nonequilibrium transport processes.

Inspired by the need to gain insight into the generation of unidirectional motion from such nonequilibrium fluctuations, several different models have been introduced. They have also been classified by Hänggi and Bartussek [1]. Among these, our interest centers on the rocking inertia ratchet system, in which the particles move in an asymmetric periodic potential subject to spatially uniform time-periodic forces of zero average. Some authors (see Refs. [5-8]), studied the nonstochastic deterministic case, which allows for the possibility of obtaining chaotic as well as regular dynamics, while others (Refs. [9-11]) investigated the effects of noise. In some respects, this deterministically induced chaos can mimic the role of noise by triggering current flow in either direction. The import of all these results is that the role of noise should never be ignored, even for inertia ratchets. Thus, the transport properties are strongly dependent on the fluctuations, chaos, and periodicity of the trajectories, all of which may coexist.

*u.vincent@lancaster.ac.uk
The interaction between two or more particles diffusing in a ratchet potential can result in collective dynamics such as synchronization, transport enhancement, clustering, current inversion, or spontaneous current flow. The possible resultant effects have been considered in several different contexts [12-28]. These studies have been motivated largely by the fact that many such systems, for example molecular motors, coexist in large numbers and do work in mutual cooperation [13]. Previous synchronization studies assumed that a pair of ratchets would interact directly via nonstochastic couplings. In some microscopic systems, however, the interaction is not direct [29-36]. For instance, populations of cells in which oscillatory reactions occur (e.g., as in circadian rhythms), communicate via chemicals that diffuse in the surrounding medium [29,31,32,37-40]. Similarly, suspensions of yeast in nutrient solutions may undergo transitions to coordinated activity that are also believed to arise through communication via chemicals diffusing in the surrounding medium [35,39]. Within an environment that may be changing, the ability to communicate between cells is an absolute requirement to ensure appropriate and robust coordination of their activities at all levels of the organism. In this configuration, the collective dynamics of all the systems can in principle be adjusted simultaneously by tuning the physical characteristics of the interconnecting medium.

Moreover, all macroscopic physical systems are unavoidably subject to random fluctuations (noise). Thus, the interplay between noise and coupling in a system of interacting particles is relevant for many applications [41-44]. The effect of noise on coupled inertia ratchets has hitherto been neglected [18-28]. Consideration of noise is also significant because interesting and potentially useful phenomena may emerge. Noise can, for instance, induce and/or enhance resonances, spiking, and synchronization [43-48]. García-Álvarez et al. [46] showed that noise and (direct) coupling are often competing factors in the induction of synchronization, and their coexistence 
can inhibit the synchrony independently induced by either. Furthermore, all cell components exhibit intracellular noises owing to random births and deaths of individual molecules, as well as to environmental fluctuations [29,31,32]. Gene regulation, in particular, is an inherently noisy process involving stochastic fluctuations owing to low copy numbers of many molecules per cell and variations in the external environment. Notably, stochastic fluctuations may not only affect the dynamics of the entire system but may perhaps also be exploited by living organisms to actively facilitate certain functions, such as synchronization and communication.

In this paper, we investigate the combined effects of network size, coupling, and noise on dynamics and transport when inertia ratchet system are coupled indirectly by means of a common stochastic dynamical environment. We will show that the synchrony of a network of particles in a ratchet potential is strongly dependent on the network size and is attained much faster for large networks. For small network size, noise enhances synchronization in the weakly coupled regime; for larger network size, the particles are only weakly synchronized. Similarly, in the strongly coupled regime, particles with small network size are weakly synchronized; whereas the synchronization is strong and robust against noise when the network size is large. While the underlying dynamics of the periodic solutions show resonance-like oscillations triggered by symmetry-breaking bifurcations for small networks, dynamics stabilization of the chaotic orbits to period orbits of different periodicity as well as quenching of irregular current fluctuations are also reported. For larger network size, the particles move with increasing speed in the ratchet potential. We will show that the size of the network, as well as its noisy environment, can significantly enhance the performance of an inertia ratchet system. The rest of the paper is organized as follows. In Sec. II, we describe the model. The stability of the collective state is analyzed in Sec. III. In Sec. IV, numerical results are presented and discussed, including the effects of the environment on chaos and the transport properties. We summarize the main results and draw conclusions in Sec. V.

\section{NETWORK COUPLED RATCHETS}

We consider a system of $N$ periodically driven particles moving in an asymmetric potential and interacting with a common noisy dynamical environment. The dimensionless inertial dynamics is governed by the following equation of motion,

$$
\begin{aligned}
& \ddot{x_{i}}=-\lambda \dot{x_{i}}-\frac{d v\left(x_{i}\right)}{d x_{i}}+F_{0} \cos (\omega t)+k_{i}\left(\varphi_{p}-x_{i}\right), \\
& \dot{\varphi_{p}}=\sum_{i=1}^{N} k_{i}\left(x_{i}-\varphi_{p}\right)+\xi(t) \quad(i=1,2, \ldots, N)
\end{aligned}
$$

where $v\left(x_{i}\right)$ is the asymmetric ratchet potential, $N$ is the number of ratchets (or network size), and $F_{0}$ and $\lambda$ are the forcing strength and the damping parameter, respectively. $k_{i}$ is the coupling coefficient or control gain. The second equation represents the noisy dynamical environment with state space $\varphi_{p} . \xi(t)$ is the stochastic term-a Gaussian white noise of zero mean [i.e., $\langle\xi(t)\rangle=0$ and $\left\langle\xi(t) \xi\left(t^{\prime}\right)\right\rangle=0$ ] and correlation $\left\langle\xi(t) \xi\left(t^{\prime}\right)\right\rangle=2 D \delta\left(t-t^{\prime}\right)$, with $D$ being the intensity of the

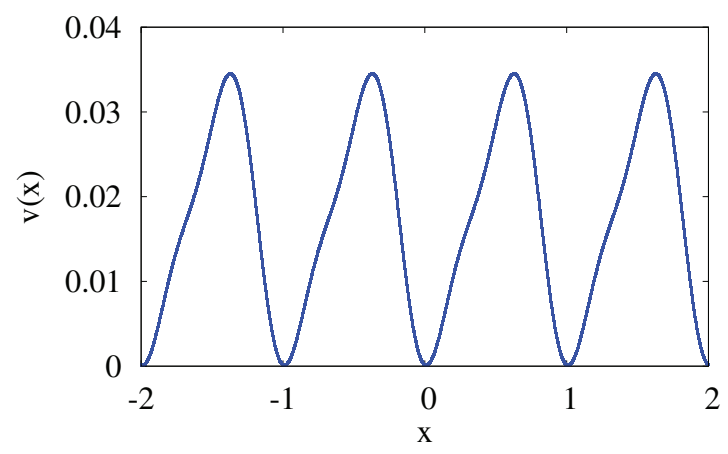

FIG. 1. (Color online) The ratchet potential Eq. (2) for $C=$ $0.0173, \delta=1.6$, and $x_{0}=-0.19$.

noise. Note that the interaction between the ratchet particles and their environment is bidirectional: each particle receives feedback from the environment, the strength of which is determined by the feedback gain $k_{i}$; and, in turn, delivers a corresponding feedback signal to the environment enabling the sustenance of the environmental activities. The period associated with the frequency $\omega$ of linear motion around the minima of the ratchet potential is used as the natural unit in which time is scaled, so that all quantities are in dimensionless units. Thus, the dimensionless potential $v\left(x_{i}\right)$ is given by [6]

$v\left(x_{i}\right)=C-\frac{1}{4 \pi^{2} \delta}\left[\sin 2 \pi\left(x_{i}-x_{0}\right)+0.25 \sin 4 \pi\left(x_{i}-x_{0}\right)\right]$,

where $C=0.0173$ and $\delta=1.6$ are fixed potential parameters. The periodic potential $v\left(x_{i}\right)$ has an infinite number of potential wells as indicated in Fig. 1. It is shifted by $x_{0}$ in order to place one of the minima at the origin.

For a single disordered (noisy) ratchet system isolated from its dynamical environment, Arizmendi et al. [9] have shown that the consequence of disorder is the appearance of current-reversal and chaotic diffusion on regular trajectories; whereas, on some chaotic trajectories, the disorder induces regular motion. In a similar study, Li [10] showed that at low noise the transport is mainly chaotic and at high noise it is mainly stochastic. Here, our system, given by Eq. (1), is such that: (i) individual ratchet units in the network are not coupled directly to each other but, rather, their interaction is provided by the stochastic dynamical environment; (ii) the environment (with state space $\varphi_{p}$ ) is a stochastic dynamical system, whereas each ratchet $\left(x_{i}\right)$ is deterministic; and (iii) when $D=0$ the global dynamics of system Eq. (1) is deterministic.

Such a coupling configuration which could, to some extent, mimic physiological processes has recently been studied in other contexts, ranging from different forms of synchronization to amplitude death [37-40,49-51]. Physiological processes are well known to be dynamic. Cells change their function in response to changes in the composition of their local environment, and the organism responds to alterations in both its internal and its external environment. Many physiological reactions are aimed at preserving a constant physical and chemical internal environment. For example, biological cells in which oscillatory reactions take place communicate via chemicals diffusing within the surrounding medium. In this process, the generation of macroscopic 
oscillations is determined by the ability of the cells to cooperate and synchronize with each other. Alternatively, as we consider here, the oscillations may arise when each of the systems synchronizes separately with their common environment [39].

In order to characterize the collective behavior of system Eq. (1), it is important to distinguish between the synchrony of a single unit with the environment and the synchrony (collective dynamics) of the full system with the environment. In this regard, we assume that each isolated ratchet system could, in the absence of another unit, be independently synchronized with the noisy environment such that

$$
n_{l}^{\varphi}=\frac{1}{T} \int_{0}^{T} \Delta_{\varphi, x_{i}} d t \rightarrow \epsilon, \quad(i=1,2, \ldots, N),
$$

where $\Delta_{\varphi, x_{i}}=\varphi_{p}-x_{i}, \epsilon$ is a very small constant, and $T$ is the period of the oscillation. Here, periodicity implies that there exists a $T>0$ such that $x_{i}(t+T)=x_{i}(t)$ and $\varphi_{p}(t+T)=$ $\varphi_{p}(t) \forall t \in \operatorname{Re}$. Thus, the synchronized oscillation corresponds to a periodic solution of Eq. (1), which is referred to as complete synchrony when $\epsilon=0$. This, however, depends on the strength $k$ of the interaction with the environment: not all $k$ values will lead to complete synchrony. In principle, not all the particles will be simultaneously synchronized with the environment. Therefore, we will hereafter refer to the synchrony attained by an individual unit as local synchronization. However, if all the particles are simultaneously synchronized with the environment, i.e., the local synchrony condition defined in Eq. (3) is satisfied for all $i=1,2,3 \ldots, N$, then the ratchets are also globally synchronized with each other. Thus global synchronization describes the collective behavior, which, in general, is a periodic solution satisfying

$$
x_{i}=x_{i+1}=x_{i+2}=\ldots=x_{N}=x, \quad(i=1,2, \ldots, N),
$$

such that the $n_{g}^{i}=\epsilon$, where

$$
n_{g}^{i}=\frac{1}{T} \int_{0}^{T} \sum_{i=1}^{N}\left(\Delta_{x_{i}}{ }^{2}+\Delta_{\dot{x}_{i}}{ }^{2}\right)^{1 / 2} d t,
$$

$\Delta_{x_{i}}=x_{i+1}-x_{i}, \Delta_{\dot{x}_{i}}=\dot{x}_{i+1}-\dot{x}_{i}$ is the global synchronization index. The existence of local and global synchronization implies that

$$
n_{g}=\frac{1}{T} \int_{0}^{T} \Delta_{\varphi, x} d t \rightarrow \epsilon,
$$

$\Delta_{\varphi, x}=\varphi_{p}-x, x=x_{i}=x_{i+1}=x_{i+2}=\ldots=x_{N}(i=1,2$, $\ldots N)$ is a state space in the three-dimensional space $\left(x, \dot{x}, \varphi_{p}\right)$, which is an invariant manifold in the original $(2 N+1)$ dimensional space $\left(x_{i}, x_{i+1}, \ldots, x_{N}, \dot{x}_{i}, \dot{x}_{i+1}, \ldots, \dot{x}_{N}, \varphi_{p}\right)$. The existence of global synchronized oscillation (i.e., collective dynamics) implies that the condition Eq. (5) is stable and that $n_{g} \rightarrow \epsilon=0$, asymptotically starting from any random set of initial conditions; it does not follow from the stability of a single unit nor from the stability of the local synchronization.

Defining the collective behavior, $x$ of the interacting network in Eq. (4) as $x=\sum_{i=1}^{N} x_{i}$, the system Eq. (1) can then be written as:

$$
\begin{aligned}
\ddot{x} & =-\lambda \dot{x}-\frac{d v(x)}{d x}+F_{0} \cos (\omega t)+k\left(\varphi_{p}-x\right), \\
\dot{\varphi_{p}} & =N k\left(x-\varphi_{p}\right)+\xi(t),
\end{aligned}
$$

where $v(x)$ is derivable from Eq. (2) and all the parameters are as in Eqs. (1) and (2).

\section{STABILITY OF THE COLLECTIVE STATE IN THE DETERMINISTIC LIMIT}

To facilitate the stability analysis, consider the following Langevin equation for general Brownian particles interacting with a stochastic environment like that of Eq. (1):

$$
\ddot{\mathbf{x}}_{i}=f^{\prime}\left(\mathbf{x}_{i}\right)-\mathbf{k}_{i}\left[f\left(\mathbf{x}_{i}\right)+\mathbf{k}_{i} \Delta_{\varphi, \mathbf{x}_{i}}+\sum_{i=1}^{N} \mathbf{k}_{i} \Delta_{\varphi, \mathbf{x}_{i}}-\xi(t)\right],
$$

where $f\left(\mathbf{x}_{i}\right)$ is a nonlinear function of the system vector state space $\mathbf{x}_{i}$. Accordingly, the global dynamics Eq. (7), assuming that the coupling coefficient $\mathbf{k}_{i}(i=1,2, \ldots N)$ takes a single value $\mathbf{k}$ is expressible as

$$
\ddot{x}=f^{\prime}(\mathbf{x})-\mathbf{k}\left[f(\mathbf{x})+\mathbf{k} \Delta_{\varphi, x}+N \mathbf{k} \Delta_{\varphi, x}-\xi(t)\right] .
$$

We examine the stability of the collective dynamics, Eq. (4), by analyzing the stability conditions for the equation of motion, Eq. (9). We aim at determining the dependence of the stability of the in-phase-mode (i.e., a periodic solution) on the system size $(N)$ and the coupling strength $(k)$. In the limit of small noise $(D \rightarrow 0)$, the stochastic dynamics may be approximated by deterministic dynamics. Thus, neglecting the noise term, we first decompose system Eq. (9) as follows:

$$
\begin{aligned}
\dot{x} & =f(x)+k \Delta_{\varphi, x}=f(x)+k \varphi_{p}-k x, \\
\dot{\varphi}_{p} & =-N k \Delta_{\varphi, x}=-N k\left(\varphi_{p}-x\right) .
\end{aligned}
$$

If $\xi_{0}$ and $\eta_{0}$ are the disturbances of the motion along the $x$ and $\varphi$ directions, respectively, the linearized equation of motion for system Eq. (10) is given by

$$
\dot{\xi}_{0}=\left[f^{\prime}(x)-k\right] \xi_{0}+k \eta_{0}, \quad \dot{\eta}_{0}=-N k\left(\eta_{0}-\xi_{0}\right) .
$$

The synchronized dynamics corresponds to the stability of the fixed point $(0,0)$ of Eq. (11); in which case all the Lyapunov exponents will be negative. Since the attractor of the system is bounded in $x$ and the periodic function in $v(x)$ can be approximated by the mean-value theorem [27], it is convenient to assume that the average value of $f^{\prime}(\mathbf{x})$ is constant (say, $\beta)$. We remark that this type of approximation has been employed successfully by previous authors and has proved to give reasonable predictions and descriptions of the global features of the phase diagrams of chaotic systems [40,52]. Eliminating $\eta_{0}$ from Eq. (11), we obtain the characteristic equation for $\xi_{0}$ as follows:

$$
\ddot{\xi}_{0}=[\beta-(1+N) k] \dot{\xi}_{0}+N k \beta \xi_{0} .
$$

In the limit $N \rightarrow \infty, N \gg 1$, so that $N+1 \approx N$. By assuming the solution of Eq. (12) in the form $A e^{m t}$, we obtain

$$
m=\frac{\beta-N k \pm \sqrt{(\beta-N k)^{2}+4 N k \beta}}{2} .
$$

The stability condition can be obtained according to the nature of the solutions of Eq. (13), from which we obtain two real solutions, namely $m_{1}=\beta$ and $m_{2}=-N k$. The first solution, $m_{1}$, depends on the nature of the nonlinearity while the second, 
$m_{2}$, depends on the network size, $N$, and strength of the interaction with the environment.

\section{RESULTS AND DISCUSSION}

Let us consider first the dynamics of a single-ratchet system coupled to the environment. We show in Fig. 2 the local synchronization index of a single unit in a network whose environment is defined by Eq. (3), for different noise levels. For the deterministic case $D=0, \eta_{l}^{\varphi}(k)$ shows a smooth resonance curve with two maxima (0.67 and 0.683$)$, located at $k=0.21$ and 0.37 , respectively (see magnified inset). The appearance of two peaks may be attributed to the fact that the system oscillates, first, near its primary resonance for the set of parameters used; the same resonance peak has also been obtained [53] for a different set of parameters. Second, however, the interaction of the system with its environment leads to oscillations away from the primary resonance via a symmetry-breaking bifurcation as shown in Fig. 2. This symmetry-breaking phenomenon could be associated with the breaking of the symmetry of the nonequilibrium potential given by Eq. (2) [54]. In Fig. 2, the resonance bifurcation of the local maxima of $x$ corresponding to the first peak of $\eta_{l}^{\varphi}(k)$ is immediately followed by the symmetry-breaking bifurcation of the periodic orbits occurring near the second peak of $\eta_{l}^{\varphi}(k)$. For small noise intensities (typically $D \leqslant$ 0.0001 ), the stochastic effect is not strongly pronounced so that stochastic dynamics could readily be approximated by its deterministic dynamics - in agreement with our earlier assumption. However, strong noise intensities can lead to more complicated behavior as seen in Fig. 2 for $D=0.05$. The complexity increases considerably with strongly erratic behavior occurring as the noise strength $D$ increases.

We now turn to the collective behavior of $N>1$-coupledratchet system, considering first the noiseless case $(D=0)$. We plot $\eta_{g}$ as a function of coupling strength $k$ in Fig. 3, showing the global synchronization index of coupled ratchets in the network with the environment for different network sizes. On the one hand, we find that for a given network

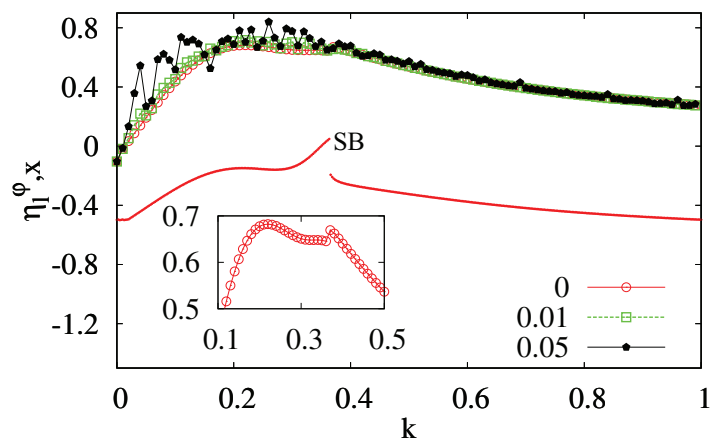

FIG. 2. (Color online) Local synchronization index $\eta_{l}^{\varphi}$ and the corresponding bifurcation of the orbits $x$ as function of coupling strength $k$ showing the local convergence of a single unit in the network to the equilibrium state for different noise levels $D=0,0.01$, and 0.05 . Red dotted points is the bifurcation curve, SB being the symmetry bifurcation point. The inset is a magnification of the region near the maximum for the $D=0$. The other parameters are $\lambda=0.75$, $F_{0}=0.5$, and $\omega=0.5$.

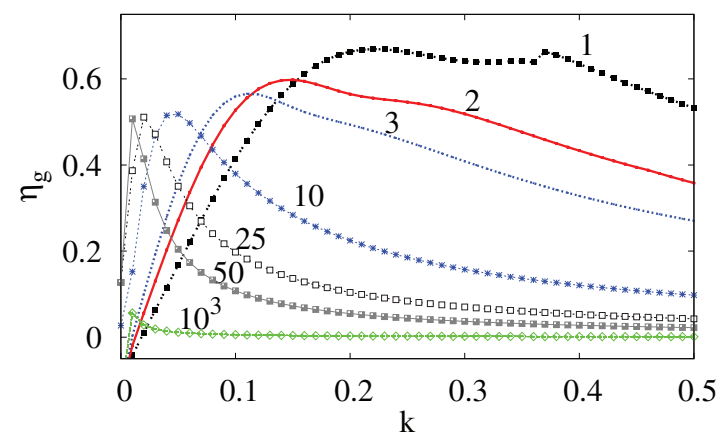

FIG. 3. (Color online) Global synchronization index $\eta_{g}$ as a function of coupling strength $k$ in the absence of noise, $D=0$, showing the global convergence of the coupled ratchets in the network to the equilibrium state for indicated network-sizes, $N=$ $1,2,3,10,25,50$, and 1000 . The other parameters are $\lambda=0.75$, $F_{0}=0.5$, and $\omega=0.5$.

size $N, \eta_{g}$ always passes through a maximum with variation of the coupling strength, i.e., a coupling-induced resonance. Also notable is the disappearance of the second peak that exists in the case of a single unit, for all $N>1$. Although the peaks do not differ significantly for $N \leqslant 100$, for $N \geqslant$ 500 there is a significant depression of the peak and a collapse of the spread-resulting in faster convergence to the synchronization manifold. When the network size is small, $k$ must be sufficiently large in order for the oscillators to attain a stable collective state. We have computed $\eta_{g}$ for larger network sizes $(N=2000,3000,5000,10000)$ and found that the collective state remains stable for large system size.

Similarly, for a given coupling strength $k, \eta_{g}$ goes through a maximum as the network size changes, i.e., network-size resonance. This is illustrated in Fig. 4. It shows that the synchronization manifold can be reached faster with larger network size than with smaller network sizes, even for weak coupling strength. In real systems, noise is unavoidably present and is, therefore, an important factor; and the collective behavior of the coupled system may depend strongly on the noise intensity. Figure 5 illustrates the influence of moderate noise level on the collective dynamics. For small network size, the effect of noise is more pronounced and especially in the

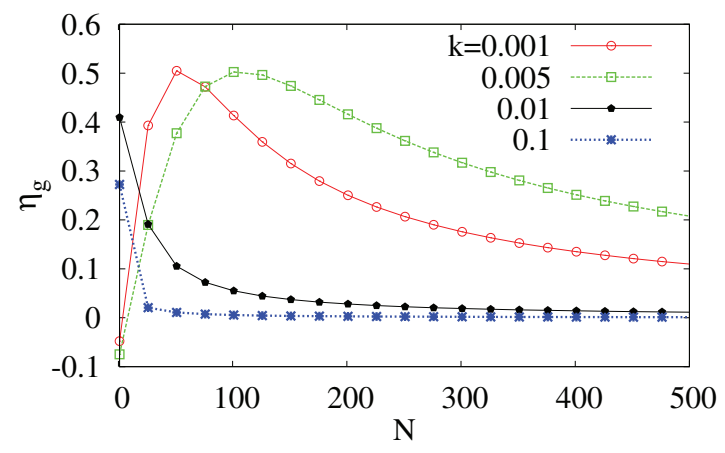

FIG. 4. (Color online) Global synchronization index $\eta_{g}$ as a function of the network-size $N$ for the noiseless case, $D=0$, showing global convergence of coupled ratchets in the network to the equilibrium state, for different coupling strengths $k$. The other parameters are $\lambda=0.75, F_{0}=0.5$, and $\omega=0.5$. 


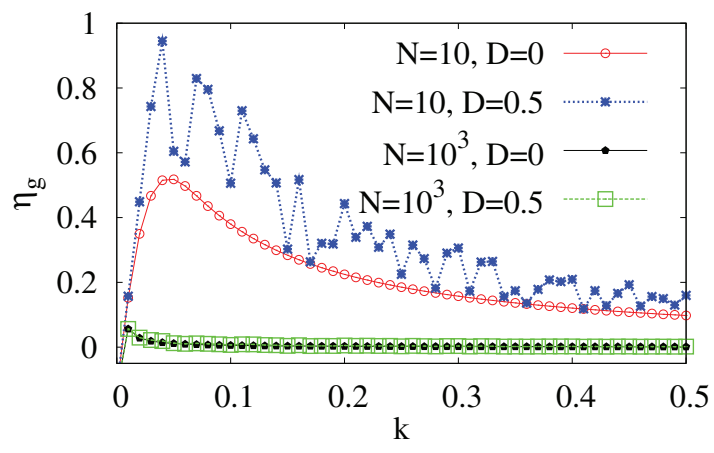

FIG. 5. (Color online) Effect of noise of intensity $D$ on the global synchronization index $\eta_{g}$ as function of $k$ for small and large network sizes $N$. The parameters are as in Fig. 3. Faster convergence is achieved with larger system size.

weak and moderate coupling regime, whereas the dynamics is robust against noise for system with large network size.

A global view of this system can conveniently be captured in a two-parameter space plot. We have performed detailed numerical simulations with $k$ and $D$ both as functions of $\eta_{g}$, scanning a wide range of different values of $k$ and $D$ for which $\eta_{g}$ takes on values between 0 and 1 ; and we have done so for different network sizes. Inside the $k-D$ parameter plane shown in Fig. 6, the region of complete synchronization $(\epsilon \leqslant$ 0.005 ) is denoted by white; while the nearly complete (weak) synchrony region is denoted by blue (dark) $(0.005 \leqslant \epsilon \leqslant$ 0.05 ). Elsewhere, the system is considered to be either weakly synchronized or completely de-synchronized. Notably, as the network size increases, the complete synchronization region in $k-D$ parameter space enlarges to the right and diminishes to the left. For small network size, increasing the noise level enhances synchronization in the weakly coupled regime [Fig. 6(a)], whereas with large network size, weak synchronization is observed for increasing noise intensity [Fig. 6(d)]. In the strongly coupled regime, and with small network size, the
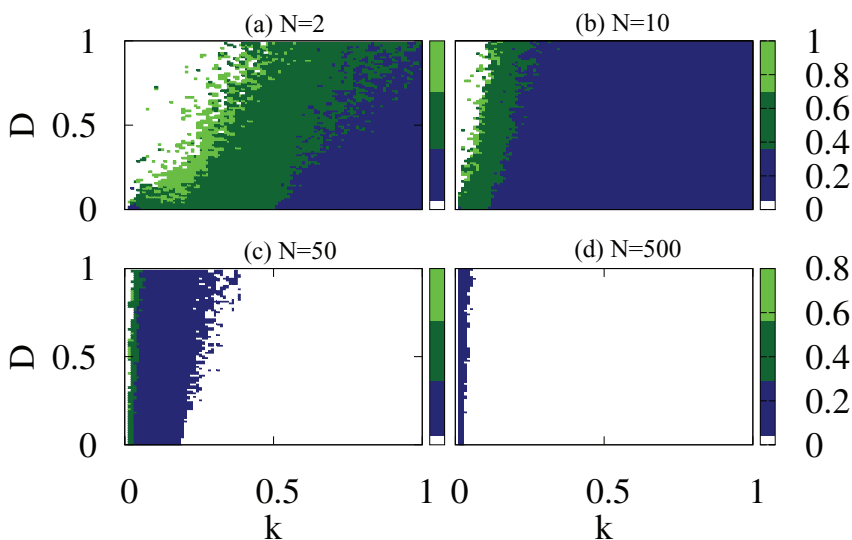

FIG. 6. (Color online) Two-parameter plot of $\eta_{g}$ as a function of coupling strength $k$ and noise intensity $D$ showing regions of full synchronization (white, $\epsilon \leqslant 0.005$ to desynchronization (green/light grey) with increasing network size, $N$. For other regions [blue (dark) and dark green (grey)], the system is either weakly synchronized or completely desynchronized. The other parameters are $\lambda=0.75$, $F_{0}=0.5$, and $\omega=0.5$. system is weakly synchronized [Fig. 6(a)]; while for large networks, strong synchronization occurs regardless of the noise intensity [Fig. 6(d)].

The relationship between bifurcations and current reversals is a topic of central importance in inertia ratchet systems because it reveals the connection between their statistical and dynamical properties. To complete our analysis, we examine the underlying bifurcations associated with the dynamics of the network for different network sizes. We use the coupling strength as the bifurcation parameter. Transport is quantified by the current $J$, which in turn is defined as the time average of the average velocity over an ensemble of initial conditions. Over a fixed time, the current in system Eq. (7) is defined $[6,8]$ as

$$
J=\frac{1}{n-n_{c}} \frac{1}{M} \sum_{l=n_{c}}^{n} \sum_{j=1}^{M} \dot{x}^{(j)}\left(t_{l}\right),
$$

where $M$ is the total number of trajectories, $t_{l}$ the observation time, and $n$ the total number of observations. This gives the average velocity, which is then further time-averaged over the number of observations $n-n_{c}$. Some transient effects are cut off in order to ensure that a converged current $n_{c}$ is obtained [8].

First, we consider the bifurcation of the periodic orbits by using the same set of parameters as before. Figures 7(a) and 7(b) illustrate, respectively, the bifurcations of the local maxima of $x$ and the corresponding current $J$, showing environment-induced directed transport for small network sizes. Although the second resonance peak in Fig. 3 appears to coincide with the $k$ value at which the symmetry-breaking bifurcation and the sudden current changes take place in Fig. 7(b), current-reversal does not accompany this bifurcation. Moreover, the consequence of the collective dynamics of a large network is the quenching and stabilization of the ensemble current as illustrated in Fig. 7(c).

By choosing the parameters to lie in the chaotic zone, namely $\lambda=0.1, F_{0}=0.0892845$, and $\omega=0.67$, as in Refs. [6,8], different scenarios are found. Figure 8 shows the bifurcation diagram for $N=1,2,5$, and 50. $N=1$ [Fig. 8(a)] corresponds to a single-ratchet system interacting with the environment. As the strength of the coupling progressively

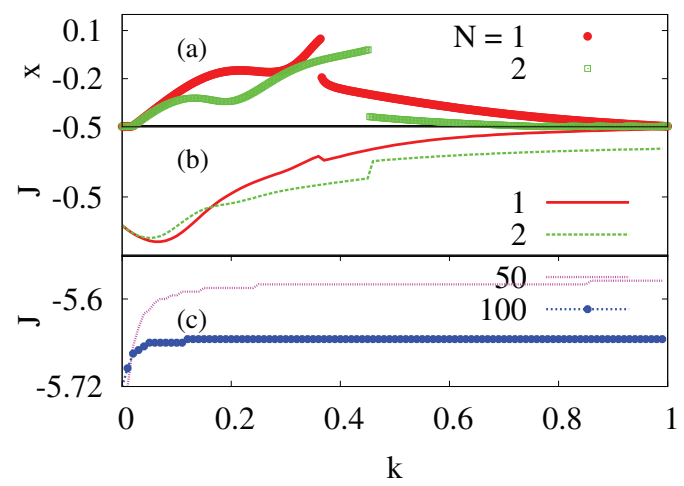

FIG. 7. (Color online) Bifurcation and current (in the units of $10^{3}$ ) as functions of the coupling strength $k$ for different network sizes, $N$. The other parameters are $\lambda=0.75, F_{0}=0.5$, and $\omega=0.5$. (a) Bifurcation of the local maxima of $x$. (b) The corresponding currents $J$. (c) Currents for large networks. 


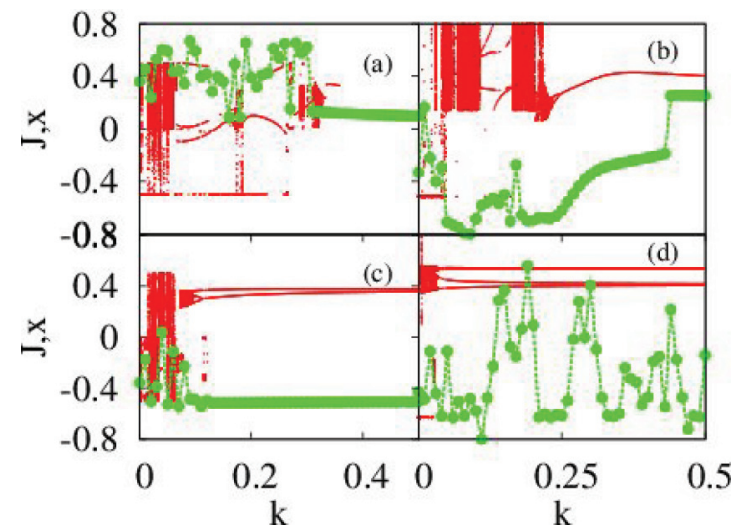

FIG. 8. (Color online) Bifurcation and current (in the units of $10^{3}$ ) as functions of the coupling strength for different network size. (a) $N=1$, (b) $N=2$, (c) $N=5$, and (d) $N=50$. the other parameters are $\lambda=0.1, F_{0}=0.0892845$, and $\omega=0.67$. Dotted points [red (dark)] are the bifurcation points $x$, while line points [green (gray)] are the currents $J$.

increases, the chaotic attractor undergoes alternating bifurcational changes: chaotic attractor $\rightarrow$ period -3 attractor $\rightarrow$ chaotic attractor $\rightarrow$ period-2 attractor $\rightarrow$ chaotic attractor and, finally, around $k \approx 0.325$, a period-doubling bifurcation takes place leading to current stabilization. Note that the current for a single particle is positive and does not change direction for any $k$ value. However, it undergoes abrupt changes near the bifurcation points as $k$ increases. For the case of the two-ratchet system $(N=2)$ shown in Fig. 8(b), a different bifurcation sequence is found. It too leads to current stabilization and it occurs within a large periodic window where symmetry breaking is found. In this case, $J$ is in general negative for nearly all $k$. However, at $k=0.43$, a currentreversal takes place in which the current is stabilized, taking on positive values for $k>0.43$. For $N=5$ and 10 , we observe entirely negative transport. Generally, with small network sizes, the attractor is stabilized into periodic orbits of lower periodicity, the corresponding effect being the stabilization of irregular fluctuations of the current-implying that small networks maximize the dynamical environment for efficient stabilization of transport. As $N$ becomes larger, as in Fig. 8(d), for instance, orbits of higher periodicity are born and these may coexist with each other and/or with chaotic attractors. Here, the current oscillates erratically and the direction is independent of the changes in bifurcation structure.

\section{CONCLUDING REMARKS}

In summary, we have investigated the collective dynamics of a network of inertia ratchets interacting indirectly through a stochastic dynamical environment. This kind of interaction occurs in physiological reaction processes aimed at preserving a constant physical and chemical internal environment by means of oscillatory reactions: communication occurs via chemicals diffusing within the surrounding medium. Specifically, biological cells change their function in response to changes in the composition of their local environment, and the organism responds to alterations in both its internal and external environment. In this process, the generation of macroscopic oscillations is determined by the ability of the cells to cooperate (collectively) and globally synchronize with each other and with their common environment. An understanding of the mechanisms associated with the interaction of living systems with their environment and the stability of the collective state (if attainable) is of both theoretical and experimental importance. The conditions for the stability of the collective state in the deterministic limit has been obtained in this paper for the stochastically coupled ratchet system: we find that the number $N$ of ratchets in the network and the strength $k$ of their interaction with the environment play key roles in the global synchronization process. It was also shown that the synchrony of the network is strongly dependent on the network size and is attained much faster when the network size is large. By increasing the noise level in the weakly coupled regime, synchronization quality is enhanced for small network size, while ratchets of large network size are weakly synchronized. Similarly, in the strongly coupled regime, coupled ratchets with small network size are weakly synchronized in comparison with larger networks that achieve strong and robust synchrony against noise. Moreover, the underlying dynamics of the periodic solutions shows resonance-like oscillations and directed transport triggered by symmetry-breaking bifurcation for small networks; while in the chaotic regime, the current becomes largely dominated by transport due to periodic orbits in a sequence of controlled bifurcations leading to stabilization by maximizing the dynamical environment for efficient transport control. However, for larger network, resonancelike oscillations of the periodic orbits disappear leading to quenching of transport fluctuations; while in the chaotic state, the current oscillates erratically and its stabilization becomes difficult. Our results show that the intrinsic properties of an environment might play a constructive role, suggesting that natural systems may profit from the interaction with their environment in order to facilitate and optimize the response to an external stimulus. Conversely, increasing the number of elements in a small ensemble of coupled biological elements to the optimum can significantly improve the sensitivity and collective effect, while changing its connectivity and/or coupling strength can also optimize its performance.

\section{ACKNOWLEDGMENTS}

U.E.V. is supported by the British Academy, the Royal Academy of Engineering, and the Royal Society of London through a Newton International Fellowship. We thank the reviewers for their constructive comments and suggestions.
[1] P. Hänggi and R. Bartussek, in Nonlinear Physics of Complex Systems: Current Status and Future (Lecture Notes in Physics), edited by J. Parisi, S. C. Muller, and W. Zimmermann (Springer, Berlin, 1996), Vol. 476, p. 294.
[2] R. D. Astumian, Science 276, 917 (1997).

[3] F. Jülicher, A. Ajdari, and J. Prost, Rev. Mod. Phys. 69, 1269 (1997).

[4] P. Reimann, M. Grifoni, and P. Hänggi, Phys. Rev. Lett. 79, 10 (1997). 
[5] P. Jung, J. G. Kissner, and P. Hänggi, Phys. Rev. Lett. 76, 3436 (1996).

[6] J. L. Mateos, Phys. Rev. Lett. 84, 258 (2000).

[7] W.-S. Son, I. Kim, Y. J. Park, and C. M. Kim, Phys. Rev. E 68 , 067201 (2003).

[8] A. Kenfack, S. M. Sweetnam, and A. K. Pattanayak, Phys. Rev. E 75, 056215 (2007).

[9] C. M. Arizmendi, F. Family, and A. L. Salas-Brito, Phys. Rev. E 63, 061104 (2001).

[10] J.-H. Li, Phys. Rev. E 74, 011114 (2006).

[11] D. G. Luchinsky, M. J. Greenall, and P. V. E. McClintock, Phys. Lett. A 273, 316 (2000).

[12] S. Cilla, F. Falo, and L. M. Floria, Phys. Rev. E 63, 031110 (2001).

[13] B. Alberts, A. Johnson, J. Lewis, and M. Raff, The Molecular Biology of Cell (Garland, New York, 2002).

[14] H. Goko and A. Igarashi, Phys. Rev. E 71, 061108 (2005).

[15] H. Chen, Q. Wang, and Z. Zheng, Phys. Rev. E 71, 031102 (2005).

[16] A. J. Fendrik, L. Romanelli, and R. P. J. Perazzo, Physica A 368, 7 (2006).

[17] H. Y. Wang and J. D. Bao, Physica A 374, 33 (2007).

[18] U. E. Vincent, A. N. Njah, O. Akinlade, and A. R. T. Solarin, Chaos 14, 1018 (2004).

[19] U. E. Vincent, A. Kenfack, A. N. Njah, and O. Akinlade, Phys. Rev. E 72, 056213 (2005).

[20] M. Kostur, P. Hänggi, P. Talkner, and J. L. Mateos, Phys. Rev. E 72, 036210 (2005).

[21] U. E. Vincent and J. A. Laoye, Phys. Lett. A 363, 91 (2007).

[22] U. E. Vincent and J. A. Laoye, Physica A 384, 230 (2007).

[23] P. L. Lu, Y. Yang, and L. Huang, Phys. Lett. A 372, 3978 (2008).

[24] J. L. Mateos and F. R. Alatriste, Chaos 18, 043125 (2008).

[25] D. G. Zarlenga, H. A. Larrondo, C. M. Arizmendi, and F. Family, Phys. Rev. E 80, 011127 (2009).

[26] S. Y. Xu, Y. Yang, and L. Song, Phys. Lett. A 373, 2226 (2009).

[27] U. E. Vincent, O. I. Olusola, D. Mayer, and P. V. E. McClintock, J. Phys. A: Math Theor 43, 165101 (2010).

[28] U. E. Vincent, A. Kenfack, D. V. Senthilkumar, D. Mayer, and J. Kurths, Phys. Rev. E 82, 046208 (2010).

[29] M. B. Elowitz, A. J. Levine, E. D. Siggia, and P. S. Swain, Science 297, 1129 (2002).

[30] C. T. Steele, B. D. Zivkovic, T. Siopes, and H. Underwood, Am. J. Physiol. Regul. Integrat. Comp. Physiol. 284, R208 (2003).

[31] J. Paulsson, Nature (London) 427, 415 (2004).
[32] L. Chen, R. Wang, T. Zhou, and K. Aihara, Bioinformatics 21, 2722 (2005)

[33] S. H. Strogatz, D. M. Abrams, A. McRobie, B. Eckhardt, and E. Ott, Nature (London) 438, 43 (2005).

[34] I. Fischer, R. Vicente, J. M. Buldu, M. Peil, C. R. Mirasso, M. C. Torrent, and J. Garcia-Ojalvo, Phys. Rev. Lett. 97, 123902 (2006).

[35] A. F. Taylor, M. R. Tinsley, F. Wang, Z. Huang, and K. Showalter, Science 323, 614 (2009).

[36] J. Zamora-Munt, C. Masoller, J. Garcia-Ojalvo, and R. Roy, Phys. Rev. Lett. 105, 264101 (2010).

[37] E. Camacho, R. Richard, and H. Howland, Int. J. Solids Struct. 41, 2133 (2004).

[38] K. Rompala, R. Rand, and H. Howland, Comm. Nonl. Sc. Numer. Simulat. 12, 794 (2007).

[39] G. Katriel, Physica D 237, 2933 (2008).

[40] V. Resmi, G. Ambika, and R. E. Amritkar, Phys. Rev. E 81, 046216 (2010).

[41] V. S. Anishchenko, V. Astakhov, A. B. Neiman, T. E. Vadivasova, and L. Schimansky-Geier, Nonlinear Dynamics of Chaotic and Stochastic Systems (Springer, Berlin, 2007).

[42] L. Schimansky-Geier, D. Abcott, A. Neiman, and C. van den Broeck, eds., Noise in Complex and Stochastic Systems, Vol. 5114 of Proceedings of SPIE (Bellingham, New Mexico, 2003).

[43] C. Zhou, J. Kurths, I. Z. Kiss, and J. L. Hudson, Phys. Rev. Lett. 89, 014101 (2002).

[44] C. Zhou and J. Kurths, Phys. Rev. Lett. 88, 230602 (2002).

[45] A. Maritan and J. R. Banavar, Phys. Rev. Lett. 72, 1451 (1994).

[46] D. García-Álvarez, A. Bahraminasab, A. Stefanovska, and P. V. E. McClintock, Europhys. Lett. 88, 30005 (2009).

[47] A. Kenfack and K. P. Singh, Phys. Rev. E 82, 046224 (2010).

[48] D. V. Senthilkumar and J. Kurths, Eur. Phys. J. Special Topics 187, 87 (2010).

[49] V. Resmi, G. Ambika, and R. E. Amritkar, Phys. Rev. E 84, 046212 (2011).

[50] V. Resmi, G. Ambika, R. E. Amritkar, and G. Rangarajan, Phys. Rev. E 85, 046211 (2012).

[51] A. Sharma, P. R. Sharma, and M. D. Shrimali, Phys. Lett. A 376, 1562 (2012).

[52] G. Ambika and R. E. Amritkar, Phys. Rev. E 79, 056206 (2009).

[53] B. R. Nana-Nbendjo, U. E. Vincent, and P. V. E. McClintock, Int. J. Bifurcat. Chaos 22, 1250141 (2012).

[54] B. von Haeften, G. Izus, and H. S. Wio, Phys. Rev. E 72, 021101 (2005). 